\title{
Deletion of the sex-determining gene SXI1a enhances the spread of mitochondrial introns in Cryptococcus neoformans
}

\author{
Zhun Yan' ${ }^{1}$ Zhimin Li', Li Yan', Yongting Yu', Yi Cheng ${ }^{1}$, Jia Chen', Yunyun Liu', Chunsheng Gao', \\ Liangbin Zeng ${ }^{1}$, Xiangping Sun ${ }^{1}$, Litao Guo ${ }^{1}$ and Jianping $\mathrm{Xu}^{2^{*}}$ (D)
}

\begin{abstract}
Background: Homing endonuclease genes (HEGs) are widely distributed genetic elements in the mitochondrial genomes of a diversity of eukaryotes. Due to their ability to self-propagate within and between genomes, these elements can spread rapidly in populations. Whether and how such elements are controlled in genomes remains largely unknown.

Results: Here we report that the HEG-containing introns in the mitochondrial COX1 gene in Cryptococcus neoformans are mobile and that their spread in sexual crosses is influenced by mating type (MAT) a-specific homeodomain gene SXI1a. C. neoformans has two mating types, MATa and MATa. In typical crosses between strains of the two mating types, only a small portion (<7\%) of diploid fusants inherited the HEGs from the MATa parent. However, disruption of the SXI1a gene resulted in the majority (>95\%) of the diploid fusants inheriting the HEG-containing introns from the MATa parent, a frequency significantly higher than those of intronless mitochondrial genes.
\end{abstract}

Conclusions: Our results suggest that SXI1a not only determines uniparental mitochondrial inheritance but also inhibits the spread of HEG-containing introns in the mitochondrial genome in C. neoformans.

Keywords: HEG, Intron, Mating type, mtDNA inheritance

\section{Background}

Homing endonuclease genes (HEGs) are mobile genetic elements that can spread from HEG-containing $\left(\mathrm{HEG}^{+}\right)$ alleles to their cognate alleles without HEG ( $\left.\mathrm{HEG}^{-}\right)$. HEGs are typically located in introns and are broadly distributed in all three Domains of life, including in both the nuclear and organelle genomes of all major groups of eukaryotes [1]. However, despite their broad distribution, HEGs are not known to have any positive contribution to host fitness and function. Rather, their sole purpose seems to be enhancing their own spread. Consequently, HEGs have traditionally been considered selfish or parasitic genetic elements [2,3]. The rapid spread of HEGs is achieved by a process termed "homing" which involves recognition and cutting of the $\mathrm{HEG}^{-}$allele by the HEG-encoded

\footnotetext{
* Correspondence: jpxu@mcmaster.ca

${ }^{2}$ Department of Biology, McMaster University, Hamilton, ON L8S 4K1, Canada Full list of author information is available at the end of the article
}

endonuclease. The disrupted $\mathrm{HEG}^{-}$allele is then repaired via a recombination-dependent repair system using an intact $\mathrm{HEG}^{+}$allele as template. In contrast, the $\mathrm{HEG}^{+}$allele is immune to such a cleavage because the presence of HEG interrupts the recognition and cleavage site. The end result of intron homing is the insertion of HEG and its associated intron into the $\mathrm{HEG}^{-}$locus, leading to the presence of HEGs on both alleles $[4,5]$.

The high efficiency of the "homing" process in nature can be seen from the distribution patterns of HEGs in both natural populations as well as in laboratory genetic crosses $[6,7]$. In sexual crosses, HEGs are often inherited by more than $95 \%$ of progeny rather than the typical Mendelian proportion of $50 \%$ for nuclear alleles [7, 8]. With such a high transmission frequency, a HEG gene could increase its frequency from 0.001 to $0.999 \%$ in about 15 outcrossed generations in a sexual population [3]. Indeed, in a survey of the $\omega$-HEG and associated

(c) The Author(s). 2018 Open Access This article is distributed under the terms of the Creative Commons Attribution 4.0 International License (http://creativecommons.org/licenses/by/4.0/), which permits unrestricted use, distribution, and 
group I intron in the mitochondrial genomes of 20 ascomycete yeast species, Goddard et al. (1999) showed that the distribution of $\omega$-HEG was not clustered on the host phylogeny and that the phylogeny based on $\omega$-HEG sequences differed significantly from that of the host species as inferred using house-keeping genes. Their analyses suggested a cyclical model of $\omega-\mathrm{HEG}$ invasion, degeneration, and loss, followed by reinvasion etc. [3].

While HEGs are prevalent in the mitochondrial genomes of many fungal species, they are noticeably absent in the mitochondrial genomes of animals [9]. One major difference between the fungal and animal mitochondrial genomes is their modes of transmission in sexual crosses. In animals, the mitochondrial genome is inherited almost exclusively from the maternal parent [9-11]. However, in fungi, there is a diversity of patterns, ranging from strictly uniparental to biparental $[12,13]$. In addition, in an increasing number of fungal species, there are significant polymorphisms in HEG distributions among individuals within populations [14-16]. Such distribution patterns suggest that there are likely genetic mechanisms controlling the transmission of HEGs.

One hypothesis suggested that uniparental organelle inheritance might have evolved to control the spread of selfish elements in organelle genomes [10, 17-19]. Consistent with this hypothesis, in the plant fungal pathogen Ustilago maydis, uniparental mitochondrial inheritance was associated with a lower transmission efficiency of HEGs in the mitochondrial genome [20, 21]. However, HEGs in the chloroplast genome of the algae Chlamydomonas are freely transferred to the sexual zygotes despite the uniparental inheritance of the chloroplast genome [22, 23]. At present, the reasons for the different results between the two different organelles and species remain unknown.

In the basidiomycete yeast Cryptococcus neoformans species complex (CNSC), sexual mating is controlled by one locus with two mating types, mating type a (MATa) and mating type $\alpha(M A T \alpha)$. In typical crosses between strains of MATa and $M A T \alpha$, the progeny inherit their mitochondrial genome almost exclusively from the $M A T$ a parent [24]. We previously identified that a $M A T \alpha$ specific gene, SXI1 $\alpha$, plays an important role in controlling mitochondrial inheritance in CNSC. Disruption of this gene resulted in biparental mitochondrial inheritance, significant heteroplasmy, and the recovery of recombinant mitochondrial genomes [25]. Furthermore, studies have shown that the mitochondrial genomes of this species complex are small ( 24 to $33 \mathrm{~kb}$ ) and polymorphic in the distribution of HEGs and their associated introns $[14,26]$. However, the potential role of SXI1 $\alpha$ in the spread of mitochondrial HEGs has not been investigated. The small mitochondrial genome size, the naturally existing HEG distribution polymorphisms in mitochondrial genomes among strains, and the presence of a gene that is known to control uniparental mitochondrial inheritance present a unique opportunity to investigate the potential mechanism of mitochondrial HEG mobility in CNSC.

\section{Methods}

\section{Strains}

The strains used for crossing experiments are listed in Table 1. These strains are all serotype D (Cryptococcus neoformans var. neoformans) and are isogenic except at the indicated loci. The origins of strain YZX2 and of strains CHY618, CHY620, CHY647 and CHY648 were described in Yan et al. [25] and in Hull et al. [27] respectively. Briefly, YZX2 is a MATa strain with a mitochondrial genotype characteristic of serotype A strains (Cryptococcus neoformans var. grubii) at the NADH dehydrogenase subunit \#2 and \#4 (ND2 and ND4) loci [25]. The mitochondrial genome of the representative serotype A strain H99 is 24,874 bp long and contains only one intron, located in the cytochrome b (COB1) gene (GenBank accession number NC004336). Strain YZX2 is auxotrophic for adenine and contains a neomycin phosphotransferase gene (NEO) coding for G418 (geneticin) resistance [25]. In contrast, strains CHY618, CHY620, CHY647 and CHY648 were derived from the

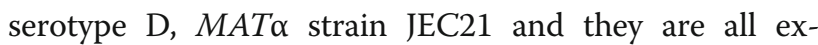
pected to have a mitochondrial genome, including intron distribution, identical to that of strain JEC21. While the complete mitochondrial genome of JEC21 is not available in public databases, it has been analyzed and reported as 33,194 bp long and contains the same number of introns at the same positions as that of strain IFM5844 [14, 26]. IFM5844 is a serotype D strain with deposited mitochondrial genome sequence through multiple GenBank accessions (AY138989, AF534132, AF534567, AF538354, AF538355, AF532780, AY560607, AY560609,

Table 1 Parental strains used in this study and their genotypes

\begin{tabular}{lll}
\hline Strain & Genotype $^{\text {a }}$ & Reference \\
\hline CHY618 & MATa ura5 mtD sxi1a::NAT & Hull et al. 2002 \\
CHY620 & MATa ura5 mtD SXI1a NAT & Hull et al. 2002 \\
CHY647 & $\begin{array}{l}\text { MATa ura5 mtD sxi1a::NAT URA5 } \\
\text { ectopic pPM8 vector }\end{array}$ & Hull et al. 2002 \\
CHY648 & $\begin{array}{l}\text { MATa ura5 mtD sxi1a::NAT URA5 } \\
\text { ectopic pPM8-SXI1a }\end{array}$ & Hull et al. 2002 \\
YZX2 & MATa ade2 mtA NEO & Yan et al. 2004
\end{tabular}

${ }^{a}$ The above five strains are isogenic except at the indicated loci. MATa: mating type a; MATa: mating type a. Strains with ura5 or ade2 auxotrophic markers require uracil or adenine respectively for growth on the minimum SD medium. $\mathrm{mtA}$ and $\mathrm{mtD}$ refer to the two parental mitochondrial genotypes distinguished by polymorphisms at loci ND2, ND4, ND5 and COX1. mtD contains one and four extra introns in ND5 and COX1 respectively compared to $\mathrm{mtA}$ in strain YZX2. For details of alleles at these three loci as well as at the COX1 locus, please see the main text, Fig. 1, Table 3, and references Xu (2002) and Yan and Xu (2003) 
and AY560611) [14]. Specifically, both strains IFM5844 and JEC21 share a total of 10 mitochondrial introns dispersed in four genes: two each in $C O B 1$ and the large subunit of the ribosomal rRNA ( $L s r R N A)$ gene, five in the cytochrome c oxidase I (COX1), and one in NADH dehydrogenase subunit 5 (ND5) $[14,26]$. Sequence analysis of the mitochondrial genomes of strains JEC21 and IFM5844 revealed that the two introns in the $C O B 1$ gene and four of the five introns in the COX1 gene contained the LAGLIDADG motif, characteristic of HEGs [14, 26]. These well-characterized mitochondrial features make CNSC an excellent model from which to investigate the potential mobility of mitochondrial HEGs and their associated introns.

For strain CHY618, the sex-determining gene SXI1 $\alpha$ was disrupted by the gene coding for NAT (nourseothricin) resistance. In contrast, strain CHY620 contains the wild-type SXI1 $\alpha$ gene but with an ectopic copy of the NAT resistance gene $[25,27]$. Thus, strain CHY620 serves as a reference control of CHY618 in sexual crosses. Strain CHY648 was derived from CHY618 where an ectopic copy of SXI1 $\alpha$ in vector pPM8 was re-introduced into CHY618. Plasmid pPM8 is a $C$. neoformans shuttle vector encoding the URA5 gene. It contained a BamHI site into which the SXI1 a gene was cloned and then transformed into strain CHY618 to derive strain CHY648 [27]. Lastly, strain CHY647 is a negative control strain of CHY648 where the empty vector pPM8 without the ectopic copy of $S X I 1 \alpha$ was re-introduced into CHY618 $[25,27]$. The genotypes of these parental strains are shown in Table 1.

\section{Mating and selection of diploid fusants}

To prepare cells for mating, all five strains were first retrieved from the $-80{ }^{\circ} \mathrm{C}$ freezer, spread onto YEPD (1\% Bacto Yeast Extract, 2\% Bacto Peptone, 2\% dextrose, 2\% agar) plates, and allowed to grow at room temperature for 2-4 days. Four pairs of strains (CHY618 x YZX2, CHY620 $x$ YZX2, CHY647 x YZX2 and CHY648 x YZX2) were then mated on V8-jiuce medium $[5 \%$ V8-vegetable juice (Campbell Soup Co.), $0.5 \mathrm{~g} / \mathrm{L} \quad \mathrm{KH}_{2} \mathrm{PO}_{4}, 4 \%$ agar and $\mathrm{pH}$ 7.2], following our previously described method [25]. After $16-20 \mathrm{~h}$ incubation, the mating mixtures were serially diluted and transferred to the minimum medium synthetic dextrose (SD) agar $[1.7 \mathrm{~g}$ Yeast Nitrogen Base without Amino Acids (DIFCO), $20 \mathrm{~g}$ Dextrose, $5 \mathrm{~g}\left(\mathrm{NH}_{4}\right)_{2} \mathrm{SO}_{4}, 20 \mathrm{~g}$ Agar, per liter] supplemented with both nourseothricin and geneticin (G418) to select for diploid fusants representing independent mating events. After 4 days of growth on the selective medium at $37^{\circ} \mathrm{C}$, individual diploid fusants were randomly picked for DNA extraction and genotyping. For each fusant, the MATa and MAT $\alpha$-specific primer pairs at the STE12a and STE12 $\alpha$ genes were respectively used to confirm that the fusants were heterozygous at the mating type locus, following protocols described in Yan et al. [28].
The confirmed fusants were then genotyped at various loci in their mitochondrial genomes, as described below.

\section{Identification of mitochondrial genotypes}

The mitochondrial genotypes of the parental strains and their diploid fusants were determined by PCR or PCR-restriction fragment length polymorphisms (PCR-RFLP) at the following marker gene loci: $\mathrm{NADH}$ dehydrogenase subunits \#2, \#4 and \#5 (ND2, ND4, and ND5), COB1, LsrRNA, and COX1. The primers used for identifying mitochondrial genotypes are listed in Table 2 . The mitochondrial genotypes at ND2 and ND4 loci were

Table 2 Primers for amplifying mtDNA fragments for genotyping in this study

\begin{tabular}{|c|c|c|}
\hline $\begin{array}{l}\text { Primer pair } \\
\text { name }\end{array}$ & Primer sequence $\left(5^{\prime} \rightarrow 3^{\prime}\right)$ & $\begin{array}{l}\text { Gene name and } \\
\text { location of primer }\end{array}$ \\
\hline ND2F & CAAGCTGCACCATTCCATA & ND2 \\
\hline $\mathrm{ND} 2 \mathrm{R}$ & CCATTAGTGGTGGTACTCC & ND2 \\
\hline ND4F & GGGAGAATTTGATTCAAGTGCAAC & ND4 \\
\hline ND4R & CATACATGGAAAGGTACTAG & ND4 \\
\hline $\mathrm{Da} 20$ & GACACTACACAAGATGCCTC & COX1 exon 1 \\
\hline Da3 & GCAATAGCATATACCATCCCG & cox1 exon 3 \\
\hline $\mathrm{Da} 22$ & CTCGAGCTTACITIACAGCAG & COX1 exon 3 \\
\hline Da19 & GTACTACTCCTGTTAGTCCTC & COX1 exon 4 \\
\hline $\mathrm{Da} 26$ & CAACGGCATACGGTGGATCTATCC & cox1 exon 4 \\
\hline Da15 & CTGTTAGATATGATGGTGTGC & COX1 exon 6 \\
\hline COB1F & CCACAACCTATTAACATTAGCTACGC & COB1 exon 1 \\
\hline COB1R & CGTCTCCATCTACAAAGCCAGCAAAC & COB1 Intron 2 \\
\hline ND5F & CTATTGGTGTTACAGGAGCTCAC & ND5 \\
\hline ND5R & GAGCCTTCATACCTGCCTTATTTGC & ND5 \\
\hline LsrRNAF & CAGCAGAACCCTTCCCAGC & Upstream LsrRNA \\
\hline LsrRNAR & CСTCCACTGTCTCATGCGG & LsrRNA exon 3 \\
\hline Cox1F & TGTCTGGAGCTGGTAACCAAT & cox1 exon 1 \\
\hline Cox1R & AAGAGGTGTTCATATAGAACTGG & COX1 exon 1 \\
\hline ATP6F & GACACACTITATTACATCTCCAC & ATP6 \\
\hline ATP6R & GAAGTTCAATGGCATCCITG & ATP6 \\
\hline $\mathrm{N} 2 \mathrm{~A} 8 \mathrm{~F}$ & AACTCCCCACATAGTTATGG & ND2-ATP8ig ${ }^{a}$ \\
\hline N2A8R & СATCCСTGTTATTAATTCACT & ND2-ATP8 ig \\
\hline ATP8F & TाCAATGGGTGCTGTGTTC & ATP8-COX1ig \\
\hline ATP8R & CCGAATGTAATITGGITACCC & ATP8 \\
\hline ATP9F & CGGACTATCAGGAGCTGGAG & ATP9 \\
\hline ATP9R & TTGGTGGTCACCGTITAGAA & ATP9-COX1ig \\
\hline ND6F & ACTTGATCTCTTGCATTGG & ND6 \\
\hline ND6R & TTATGTTCGTGGTCGTAGACA & ND6 \\
\hline
\end{tabular}

${ }^{a} N D 2-A T P 8 i g$ indicates that the primer is located in the intergenic region between ND2 and ATP8

${ }^{\mathrm{b}}$ ATP8-COX1ig indicates that the primer is located in the intergenic region between ATP8 and COX1

'ATP9-COX1ig indicates that the primer is located in the intergenic region between ATP9 and COX1 
determined based on PCR-RFLP as described previously $[15,25]$. The genotypes at loci ND5, COX1, COB1 and LsrRNA were determined by PCR using primers located in intron-flanking regions as described by Toffaletti et al. [26] and Litter et al. [14]. Because the parental strains (YZX2 and the four JEC21 derivatives CHY618, CHY620, CHY647, and CHY648) did not differ at COB1 or LsrRNA loci (see Results below), we only genotyped the diploid fusants from these crosses at the following four mitochondrial loci: ND2, ND4, ND5 and COX1.

\section{DNA sequencing}

Our results showed that the HEGs in COX1 was mobile during sexual crosses (see Results below). To determine the border regions of HEG transpositions, we PCR-amplified the COX1 gene together with its flanking sequence using the following primer pairs described in Table 2: Da20 and Da3; Da22 and Da19; Da26 and Da15; N2A8F and N2A8R; ATP8F and ATP8R; ATP9F and ATP9R; and Cox1F and Cox1R. PCR products were purified using a PCR cleaning kit (Qiagen). The purified PCR products were sent for sequencing using both the forward and reverse primers at the Mobix Laboratory at McMaster University.

\section{Results}

The distribution of mitochondrial HEGs and their associated introns among the five parental strains

Based on the published mitochondrial genome sequences, we synthesized primers to confirm the presence/absence of all mitochondrial introns in the five parental strains used in this study (Table 2). Table 3 summarized the distributions of introns in these five parental strains as well as two reference strains. Our analyses indicated that strains CHY618, CHY620, CHY647 and CHY648 had all the 10 introns described for the sequenced strains JEC21 and IFM5844, including five introns in the COX1 gene, two each in the COB1 and LsrRNA genes, and one in the
ND5 gene. This result is consistent with previous reports $[14,26]$ and with our expectation because JEC21 was the progenitor strain for these four strains.

In contrast, the mitochondrial genome of the MATa parental strain YZX2 for our crosses was found to contain five introns with two each within the $C O B 1$ and LsrRNA genes and one within the COX1 gene. The intron distribution in YZX2 is thus different from those of the two published serotype A strains ( 1 intron only in serotype A strains H99 and IFO410, refs \#14 and 26) as well as the two published serotype D strains (10 introns in serotype D strains, JEC21 and IFM5844) (Fig. 1). Thus, in total, five introns absent in the mitochondrial genome of YZX2 but present in the other four strains in Table 1 (i.e. CHY618, CHY620, CHY647 and CHY648) can be used for genotyping intron mobility in this study: one located within the ND5 gene and four located within the $C O X 1$ gene. Sequence analysis showed that the COX1 intron in strain YZX2 corresponded to intron \#3 of the COX1 gene in the other four strains used in this study (Table 3). Among the four polymorphic introns within the COX1 gene, three contained HEGs (introns $\# 1$, \#2 and \#4). Together, the differences in intron distribution between the MATa YZX2 strain and the four $M A T \alpha$ strains shown in Table 1 provided us an opportunity to examine the potential mobility of introns in $N D 5$ and $C O X 1$ genes during sexual crosses.

\section{The HEG-containing introns in the COX1 gene are mobile during sexual mating}

Before examining intron distribution among progeny populations of the four crosses, we first investigated whether all the fusants selected based on auxotrophic and NAT/ NEO-resistance markers contained mating type alleles from both parental strains in all four crosses. Our analyses confirmed that all selected fusants were heterozygous at the mating type locus, representing true mating products.

Table 3 Mitochondrial intron distribution in the parental strains used in this study

\begin{tabular}{|c|c|c|c|c|c|c|c|}
\hline & JEC21 & CHY618 & CHY620 & CHY647 & CHY648 & YZX2 & $\mathrm{H} 99$ \\
\hline COB1 intron \#1 & + & + & + & + & + & + & - \\
\hline COB1 intron \#2 & + & + & + & + & + & + & + \\
\hline LsrRNA intron \#1 & + & + & + & + & + & + & - \\
\hline LsrRNA intron \#2 & + & + & + & + & + & + & - \\
\hline ND5 intron & + & + & + & + & + & - & - \\
\hline COX1 intron \#1 & + & + & + & + & + & - & - \\
\hline COX1 intron \#2 & + & + & + & + & + & - & - \\
\hline COX1 intron \#3 & + & + & + & + & + & + & - \\
\hline COX1 intron \#4 & + & + & + & + & + & - & - \\
\hline COX1 intron \#5 & + & + & + & + & + & - & - \\
\hline
\end{tabular}

Their distributions in the two common lab strains JEC21 and H99 are also described here

+ , presence of the intron; - , absence of the intron 


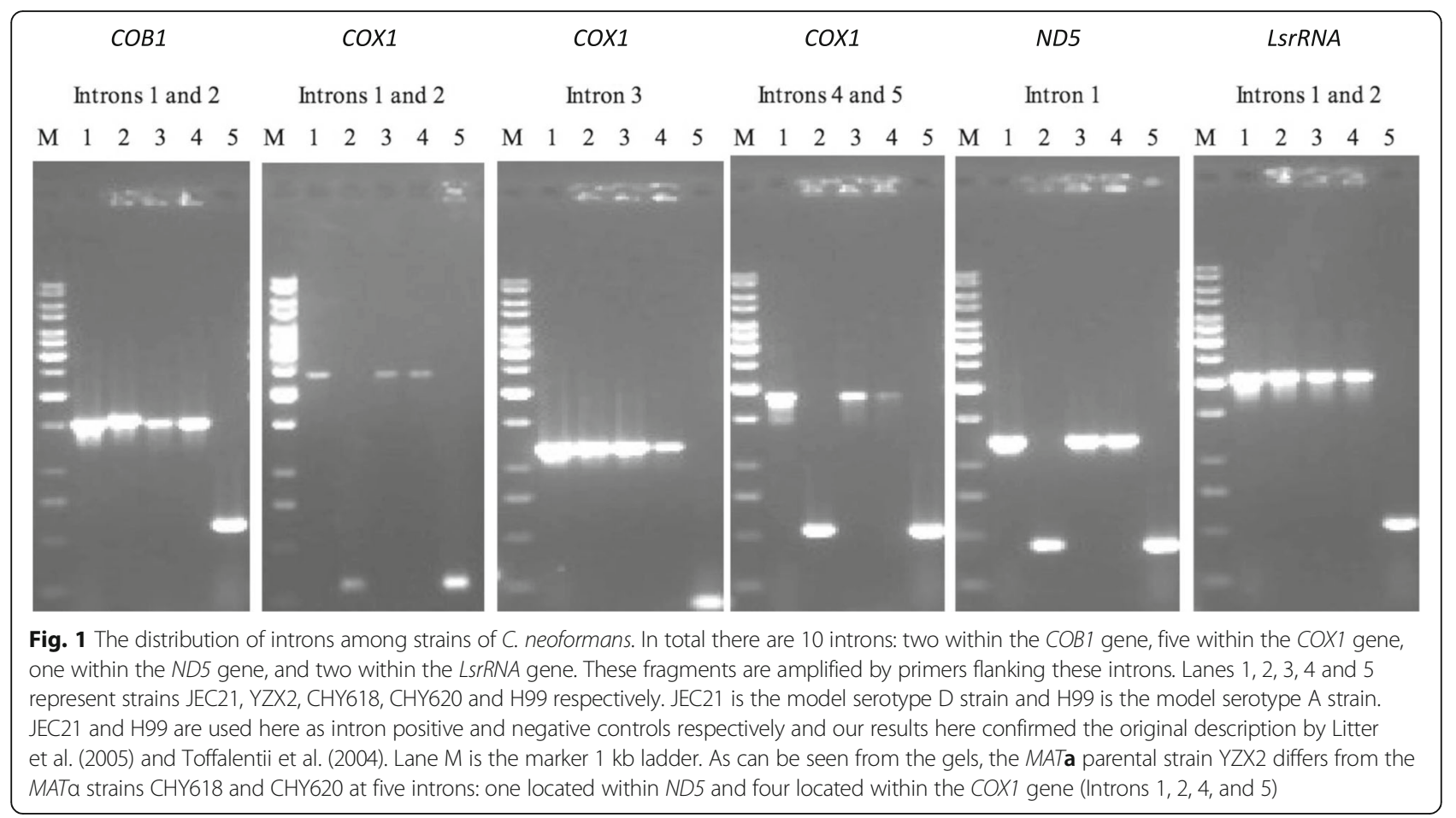

For each cross, we then used gene-specific PCR-RFLP markers at the ND2 and ND4 loci and PCR fragment length markers flanking introns in genes ND5 and COX1 to determine the mitochondrial genotype of each diploid fusant. The summary results for all four crosses are presented in Table 4.

Of the four crosses, we first analyzed diploid fusants

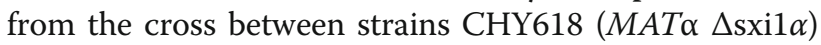
and YZX2 (MATa). Similar to results from a previous

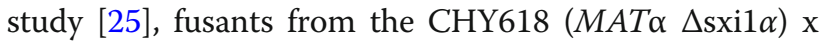
YZX2 (MATa) cross (cross \#1, Table 4) showed a diversity of mitochondrial genotypes. Indeed, significantly more progeny from this cross inherited the mitochon-

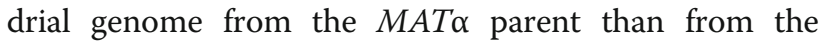
MATa parent (Chi-square statistic $=33.86 ; \mathrm{df}=1 ; p=0$, fusants with recombinant and heteroplasmic mtDNA genotypes were excluded in this test). Furthermore, there was a higher frequency of heteroplasmic or recombinant mtDNA genomes (recombinant genotype based only on alleles at ND2, ND4 and ND5 genes) than those of its reference cross with the wild-type SXI1 a gene CHY620 $x$ YZX2 (cross \#2 in Table 4) (Chi-square statistic= 12.0839; $\mathrm{df}=1 ; p=0.000509$ ). Interestingly, when we examined the progeny genotypes at the COX1 gene locus, $95.2 \%$ of the diploid fusants (100/105; excluding the 11 recombinant or heteroplasmic fusants) inherited the HEG-containing introns in the COX1 gene from the MAT $\alpha$ parent in the CHY618 x YZX2 cross (cross \#1, Table 4), significantly more than the alleles at the ND2, ND4 and ND5 loci $(\sim 75 \%)$ (Chi-square statistic $=$ $16.1345 ; \mathrm{df}=1 ; p=0.000059$; the 11 recombinant or heteroplasmic fusants were excluded from this comparison).

Table 4 Progeny mitochondrial genotypes and the number of progeny in each genotype class from four crosses

\begin{tabular}{lllllll}
\hline Cross & $\mathrm{CaNa}^{\mathrm{a}}$ & $\mathrm{CaNa}^{\mathrm{b}}$ & $\mathrm{CaNa}^{\mathrm{c}}$ & $\mathrm{CaNa}^{\mathrm{d}}$ & $\mathrm{CaNr}^{\mathrm{e}}$ & $\mathrm{CbNb}^{\mathrm{f}}$ \\
\hline 1. CHY618 X YZX2 (MATa) & 5 & 70 & 30 & 0 & 3 & 8 \\
2. CHY620 X YZX2 (MATa) & 254 & 12 & 2 & 1 & 0 & 4 \\
3. CHY648 X YZX2 (MATa) & 120 & 6 & 1 & 0 & 0 & 116 \\
4. CHY647 X YZX2 (MATa) & 4 & 43 & 30 & 0 & 3 & 11 \\
\hline
\end{tabular}

a, CaNa refers to diploid fusants having both the COX1 allele and other genetic markers originating from the MATa parent YZX2

b, CaNa refers to a diploid fusant having both the HEG-containing introns in COX1 gene and other genetic markers originating from the MATa parent

', CaNa refers to diploid fusants having the HEG-containing introns in COX1 originating from the MATa parent but other genetic markers originating from the MATa parent YZX2

d, CaNa refers to diploid fusants having the COX1 allele originating from the MATa parent but other genetic markers originating from the MATa parent e, CaNr refers to diploid fusants having the HEG-containing introns in COX1 originating from the MATa parent and recombinant mtDNA genotype with ND2 and ND5 alleles from the MATa parent and ND4 from the MATa parent

${ }_{\mathrm{f}}^{\mathrm{C}} \mathrm{CbNb}$ refers to diploid fusants having alleles from both parents at all analyzed loci 
The result clearly indicates that HEG-associated introns in COX1 gene are preferentially inherited by progeny from this cross.

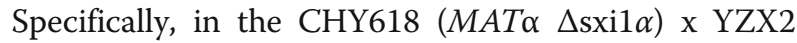
(MATa) cross (cross \#1, Table 4), 30 (of 116) diploid fusants inherited the intronless mitochondrial markers ND2, ND4 and ND5 from their MATa parent YZX2 but contained the HEG-containing COX1 introns from the

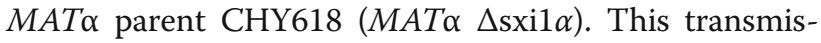
sion pattern in favor of the HEG-containing COX1 introns over the intronless markers at ND2, ND4, and ND5 is significantly more frequent than that found in the control cross CHY620 x YZX2 (cross \#2 in Table 4) where only two of the 273 diploid fusants had such a recombinant mitochondrial genotype (Chi-square statistic $=68.09 ; \mathrm{df}=1 ; p=0)$. Furthermore, there was no evidence of reciprocal recombinants among progeny from the CHY618 x YZX2 cross that contained the mtDNA markers ND2, ND4, and ND5 from the MATa parent but without any of the four HEG-containing introns in the COX1 gene from YZX2 (Table 4). The results from crosses \#1 and \#2 clearly indicate that the deletion of SXI1 $\alpha$ was associated with the spread of the HEG-associated introns in the COX1 gene.

Among the 30 progeny that inherited HEG-associated introns independent of the majority of their mitochondrial genomes, most (29/30) contained all four introns in the COX1 gene from the MATa parent. One progeny inherited only two of the four introns (introns \#1 and \#2 but not \#4 and \#5) in the COX1 gene. (Both parental strains already have intron \#3.)

Unlike the inheritance pattern of HEG-containing introns in the COX1 gene, the transmission of the ND5 intron followed the same inheritance pattern as the intronless genetic markers in the ND2 and ND4 genes.
This result suggests that the intron in ND5 is not mobile. This observation is consistent with our expectation as the intron in ND5 gene of strain CHY618 contained no LAGLIDADG motif (i.e. no HEG), and thus is not expected to move, unlike the HEG-containing introns in the COX1 gene.

\section{The spread of the HEG-associated introns is confined to the COX1 gene region}

To further examine the extent of the transposed regions within and around the COX1 gene, we sequenced the COX1 gene and its flanking regions for 10 random fusants (out of 30 mentioned above from the cross CHY618 x YZX2) that contained the transferred introns in COX1 as identified using the 6 pairs of primers listed in Table 2. Sequences from these fusants were then compared to sequences from the two parental strains. Our comparisons showed that the recombination borders for all 10 mating fusants lie close to the $5^{\prime}$ and $3^{\prime}$ ends of the COX1 gene, either within the coding region or in the adjacent flanking regions less than 48 base pairs from the translation start or end sites of the COX1 gene (Table 5, Fig. 2). In contrast, as mentioned previously, the three genes ND2, ND4 and ND5 all inherited their alleles from the MATa parent YZX2 and showed no evidence of recombination among them within this sub-population of fusants (Table 4). In addition, these three genes are widely spaced in the $C$. neoformans mitochondrial genome [14, 26]. Furthermore, the frequency of HEG-associated intron transposition (30/35) is significantly higher than that of recombination based on the three gene markers ND2, ND4 and ND5 (3/38, Table 2) (Chi-square statistic $=44.54 ; \mathrm{df}=1 ; p=0$ ). Taken together, the high frequency and high specificity of HEG-associated intron transpositions within the COX1 gene and the lack

Table 5 Locations of regions containing recombination borders in 10 diploid fusants from cross CHY618 ( $\Delta$ sxi1a) x YZX2

\begin{tabular}{|c|c|c|c|c|}
\hline Strains & Left border within this region & Location of the left border & Right border within this region & $\begin{array}{l}\text { Location of the } \\
\text { right border }\end{array}$ \\
\hline OYZ1 & $13,127 \sim 13237^{a}$ & $-\operatorname{cox} 1^{\mathrm{b}}$ & $14,440 \sim 14,786$ & $\operatorname{cox} 1^{-}$ \\
\hline OYZ2 & $13,639 \sim 13,840$ & $\operatorname{cox} 1^{d}$ & $14,440 \sim 14,786$ & $\operatorname{cox} 1-$ \\
\hline YZ150 & $13,456 \sim 13,588$ & $\operatorname{COX} 1$ & $14,440 \sim 14,786$ & $\operatorname{COX} 1-$ \\
\hline YZ151 & $13,127 \sim 13,237$ & $-\operatorname{COX} 1$ & $14,440 \sim 14,786$ & $\operatorname{coX} 1-$ \\
\hline YZ152 & $13,855 \sim 13,887$ & $\operatorname{cox} 1$ & $14,440 \sim 14,786$ & $\operatorname{coX} 1-$ \\
\hline YZ153 & $13,456 \sim 13,588$ & $\operatorname{cox} 1$ & $14,440 \sim 14,786$ & $\operatorname{cox} 1-$ \\
\hline YZ155 & $13,855 \sim 13,887$ & $\operatorname{COX} 1$ & $14,440 \sim 14,786$ & $\operatorname{coX} 1-$ \\
\hline YZ158 & $13,855 \sim 13,887$ & $\operatorname{COX} 1$ & $14,374 \sim 14,407$ & $\operatorname{cox} 1$ \\
\hline YZ159 & $13,639 \sim 13,840$ & $\operatorname{COX} 1$ & $14,297 \sim 14,374$ & $\operatorname{cox} 1$ \\
\hline YZ160 & $13,855 \sim 13,887$ & $\operatorname{COX} 1$ & $14,297 \sim 14,374$ & $\operatorname{cox} 1$ \\
\hline
\end{tabular}

${ }^{a}$ The numbers here show the location of recombination borders using the published serotype A mitochondrial genome (NC_004336) as a reference ${ }^{b}$-COX1 indicates that the left border of recombination is located either within the COX 1 gene or in the intergenic region between ATP9 and COX1 ${ }^{\mathrm{C}} \mathrm{COX} 1$ - indicates that the right border of recombination is located either within the COX1 gene or in the intergenic region between COX 1 and ATP8 ${ }^{d} \mathrm{COX} 1$ indicates that the border of recombination is located within the $\operatorname{COX} 1$ gene 


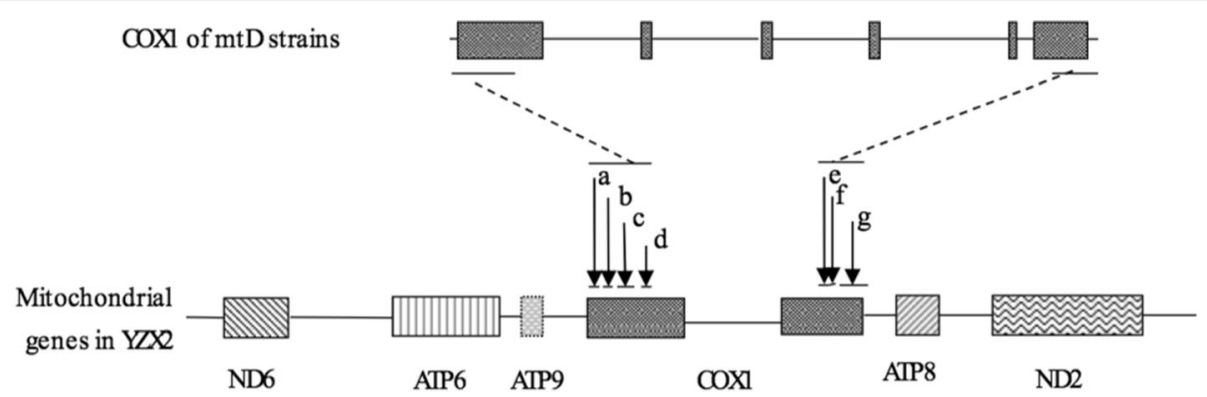

Fig. 2 Schematic representation of the mitochondrial genomic region surrounding COX1 and the borders of intron homing in 10 of the 30 diploid fusants with the CaNa mitochondrial genotypes. The arrows show the regions of integration borders. All the borders are located either within the COX1 gene or within the intergenic region between COX1 and two other genes (between ATP9 and COX1 for the left border and between COX1 and ATP8 for the right border), close to COX1 translation initiation and termination sites. Arrow "a" shows the left borders of two strains (OYZ1 and YZ151); arrow "b", the left borders of two strains (YZ150 and YZ153); arrow " $\mathrm{c}$ ", the left borders of two strains (OYZ2 and YZ159); and arrow " $\mathrm{d}$ ", the left borders of four strains (YZ152, YZ155, YZ158 and YZ160). Arrow "e" points to the right borders of two strains (YZ159 and YZ160); arrow "f", the right border of strain YZ158; arrow " $g$ ", the right border of the remaining seven strains (OYZ1, OYZ2, YZ150, YZ151, YZ152, YZ153 and YZ155)

of evidence for recombination among the other three markers in these 30 fusants suggest that the 30 novel mitochondrial genotypes were generated via homing of HEG-containing introns in the COX1 gene, and not due to mitochondrial recombination.

\section{SXI1a gene inhibits the spread of HEG-associated introns in $\mathrm{COX} 1$}

If the hypothesis that uniparental organelle inheritance evolved to prevent the spread of selfish genetic elements were correct, we would expect any gene critical for uniparental mitochondrial inheritance, like SXII $\alpha$ in this study, to play a role in the transmission of selfish elements. To test whether $S X I 1 \alpha$ can limit the transmission of HEGs in the mitochondrial genome, we analyzed three more crosses. In contrast to the cross between CHY618 and YZX2 described above, transmission of HEG-associated introns was significantly inhibited in crosses involving functional SXI1 $\alpha$ (crosses \#2 and \#3, Table 4). For example, in cross \#2 between CHY620 and YZX2, 93.4\% of the fusants (255/273) did not inherit the

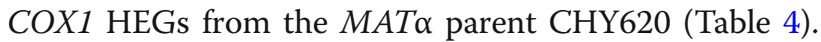
Of the remaining 18 fusants from this cross, 12 had mtDNA from only the MAT $\alpha$ parent CHY620; four had mtDNA from both parents at all loci (heteroplasmy); and two had most of the mtDNA from the MATa parent but contained the four additional COX1 introns from the

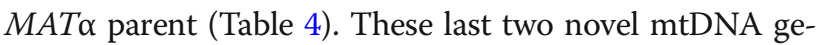
notypes were likely derived from HEG-mediated intron homing, similar to that proposed for the 30 progeny from cross \#1 described above.

Similar to cross \#2, in cross \#3 (Table 4), re-introducing the functional $S X I 1 \alpha$ allele back to CHY618 $(\Delta s x i 1 \alpha)$ restored uniparental mitochondrial inheritance and consequently also limited the spread of HEG-containing introns among the fusants. Furthermore, the results in cross \#4 showed that neither the plasmid vector pPM8 nor the selection markers influenced HEG-mediated intron transmission (Table 4, cross \#4).

Together, our results from these four crosses clearly indicated that the presence of a functional SXI1 $\alpha$ gene significantly inhibited the spread of HEG-associated introns from the intron-containing mitochondrial genome to the homologous but intronless sites in progeny mitochondrial genomes during sexual mating. The deletion of sxil $\alpha$ led to a significantly greater frequency of HEG-containing introns in $C O X 1$ gene than other genes in mating fusants. Together, our results suggest that SXI1 $\alpha$ not only controls uniparental mitochondrial inheritance but also limits the spread of the HEG-containing introns in C. neoformans (Fig. 3).

\section{Discussion}

HEGs and their associated introns have been observed in organelle genomes of many groups of organisms including Fungi, Ameobozoa, Plantae, Chromalveolata, Rhizaria, Excavata, and Metazoa [29]. However, the motility of these introns and how their transmission is controlled remain little known. In this study, we examined the mobility of mitochondrial HEG-containing introns in the human pathogenic yeast $C$. neoformans. We found that while the intron in the ND5 gene was not mobile, the four HEG-associated introns in the COX1 gene were mobile. Furthermore, we demonstrated that the $M A T \alpha$-specific homeodomain gene $S X I 1 \alpha$ influenced the mobility of the HEG-associated introns. Deletion of this gene not only disrupted uniparental mitochondrial inheritance but also enabled the mobility of the HEG-containing introns in COX1.

\section{Recombination and unidirectional transfer of HEG- containing introns in mitochondria}

In our crosses, we observed both homologous recombination and unidirectional transfer of HEG-containing introns in the mitochondrial genomes. These two processes 


\section{MATa parent MATa parent Diploid fusants}

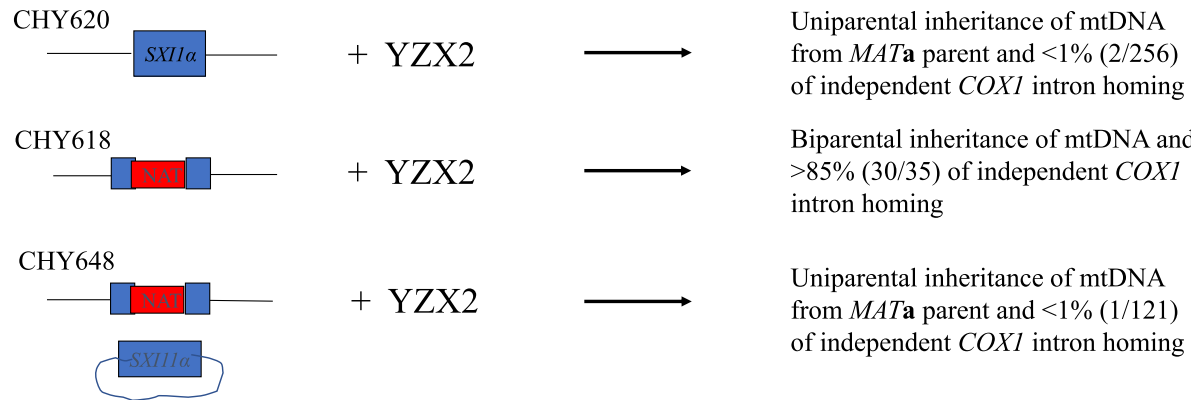

Fig. 3 Summary of the effects of SXI1a on mitochondrial DNA inheritance and mobility of HEG-containing introns in COXI gene during sexual mating in Cryptococcus neoformans. The genotypes of parental strains are shown in Table 1

have been observed in other species. For example, homologous mitochondrial genome recombination has been reported in the basidiomycete mushrooms Coprinus cinereus [30], Agrocybe aegerita [31] and Pleurotus ostreatus [32]. Similarly, independent transfers of HEG-associated introns have also been reported in other species including the baker's yeast Saccharomyces cerevisiae [7], the plant fungal pathogen $U$. maydis $[20,21]$ and the unicellular algae Chlamydomonas species [9]. In species of the model filamentous fungal genus Neurospora, mitochondrial plasmids with sequences very similar to group I introns have been found capable of independent transfer to strains without such plasmids [33]. However, the control for the above-mentioned intron transfers between organelles remains unknown.

\section{Relevance of mitochondrial intron mobility to the evolution of uniparental mtDNA inheritance}

In the great majority of sexual eukaryotes, mitochondria are inherited almost exclusively from a single parent $[9,11,34]$. Because uniparentally inherited genomes are prone to mutation accumulation [35-37], the predominance of uniparental mitochondrial inheritance has posed a serious challenge to evolutionary biologists. Indeed, many hypotheses have been proposed to explain the prevalence of uniparental mitochondrial inheritance $[10,36,38,39]$. One commonly discussed hypothesis stated that uniparental organelle inheritance evolved to prevent the spread of selfish cytoplasmic DNA such as HEG-containing introns [10, 17-19]. However, experimental evidence for this hypothesis has been lacking.

In this study, we found that the sex-determining nuclear gene SXI1 $\alpha$ was not only critical for ensuring uniparental mitochondrial inheritance, as was demonstrated previously [25], but also inhibited the spread of HEGs in the mitochondrial genome in C. neoformans. In the corn fungal pathogen, $U$. maydis, a nuclear gene $L G A 2$ was found to play a critical role in ensuring uniparental mitochondrial inheritance and in HEG transmission [20, 21]. Specifically, the absence of LGA2 led to an increased number of progeny having an HEG-containing intron in the gene coding for the mitochondrial large subunit of ribosomal RNA (LsrRNA). However, unlike in our study, intronless markers independent of the HEG-containing intron in the LsrRNA gene were not investigated in their crosses. As a result, they were unable to determine whether the increased prevalence of the mobile intron was due to the loss of control for uniparental mitochondrial inheritance or due to enhanced intron mobility. In contrast, our data clearly showed that the absence of a functional sxil $\alpha$ enhanced the spread of HEG-containing introns, independent of mitochondrial recombination and on top of biparental mitochondrial inheritance.

Our results also differ from those observed in the green algae Chlamydomonas. In Chlamydomonas, there are two types of organelles, the chloroplasts and the mitochondria. HEG transmission in the chloroplast genome of Chlamydomonas appears to be different from that in the mitochondrial genome. For HEGs in the chloroplast genome, either parent ( $m t$ - or $m t+)$ can transmit HEG to sexual progeny [22, 23]. In contrast, in the mitochondrial genome, only the $m t$ - parent transmits its mitochondrial HEG to zygotes [40]. The different HEG transmission patterns in these two organelle genomes suggest uniparental inheritance and intron mobility of these two organelle genomes are controlled by different genes and likely evolved independently. Nevertheless, data from this study and those found in $U$. maydis and Chlamydomonas all showed that uniparental mitochondrial inheritance can limit the spread of HEG-containing introns. However, only in C. neoformans has it been demonstrated that the absence of a mating 
type-specific gene sxil $\alpha$ led to the over-transmission of HEG-containing introns independent of other mitochondrial genes.

While HEG-containing introns are among the most frequently discussed selfish genetic elements, there are also other types of deleterious cytoplasmic elements including intracellular parasites as well as defective organelle genes and genomes. These genetic elements may have a replication and/or transmission advantage relative to other genes in the host cell, resulting in their over-representation in subsequent generations that may cause deleterious effects for the hosts. For example, the petite phenotype in the baker's yeast $S$. cerevisiae is typically characterized by large deletions in the mitochondrial genome and by impaired respiration [41]. In heteroplasmic cells containing both the wild-type mitochondrial genome and the petite mitochondrial genome with large deletions, the smaller mitochondrial genomes from petite mutants may exhibit a two-fold transmission advantage compared to the wild type mitochondrial genome [42]. While natural selection could eventually purge host cells with the defective mitochondrial genomes from the population, having a mechanism to prevent their transmission during sexual crosses could be highly beneficial.

\section{Conclusions and perspectives}

In this study, we identified that the mating-type $\alpha$-specific, sex-determining gene SXI1 $\alpha$ could prevent the spread of HEG-containing introns in the mitochondrial genome in C. neoformans (Fig. 3). However, we urge caution in extending our laboratory observations to natural populations. For technical reasons (i.e. crosses \#1 and \#4 are unable to produce dikaryotic hyphae, the typical mating products in CNSC, even in successful mating) and for comparative purposes among crosses, only diploid fusants were selected for analyses in this study. In contrast, mating in natural environments will likely produce dikaryotic hyphae and not diploid yeast cells. The distributions and interactions between parental mitochondria in cytoplasm could differ between dikaryotic hyphae and diploid yeast fusants. Furthermore, though diploid fusants have been reported in $C$. neoformans [28, 43], the genetic stability of the diploid fusants is currently not known. Further analyses of the distributions of HEG-containing introns among environmental and clinical strains are needed in order to understand how HEG-containing introns may have spread in nature.

SXI1 $\alpha$ is a transcription factor and can influence the expressions of many genes [44]. Previous studies have demonstrated that MAT $\alpha$ is the dominant mating type in both environmental and clinical populations of $C$. neoformans and that strain JEC21 with the MATa mating type is more virulent than its isogenic MATa partner
JEC20 $[28,43]$. In C. neoformans, the mating type loci are relatively complex, $\sim 100 \mathrm{~kb}$ in length and encoding $\sim 20$ or more genes at each of the MATa and $M A T \alpha$ loci. The SXI1 $\alpha$ gene is among the genes located within the MAT $\alpha$ locus. As a transcription factor, SXI1 $\alpha$ likely controls the spread of HEG introns indirectly through regulating the expressions of other genes. Further investigations are needed in order to identify its downstream targets and the molecular processes involved in controlling intron homing.

Regardless of the molecular mechanisms governing intron mobility, homing endonucleases have shown significant potential in genome editing and are now used in the fields of agriculture and human health [45]. For example, because HEG can spread quickly in populations, HEGs have been tested for controlling the hosts of malaria parasites, mosquitoes [46]. In our study, four of the five introns in the COX1 gene of strain CHY618 contained HEGs. Protein sequences of these HEGs in $C$. neoformans differ from other reported HEGs, with the best match to a homing endonuclease found in the mitochondrial genome of watermelon $(71 \%$ amino acid sequence identity, uniprot $\mathrm{B} 4 \mathrm{XPH} 0$ ). At present, we do not know which of the HEGs within the COX1 gene initiated the intron homing process nor which HEG has the highest activity. Knockout or over-expression of each of the four HEGs separately might allow us to pinpoint the functional significance of individual HEGs in COX1 and their recognition sequences [45, 47]. Such knowledge could help us understand the molecular processes of intron transmission in fungi, their potential roles in fungal pathogenesis, and their putative applications in genome editing.

\section{Abbreviations}

MATa: Mating type a; CNSC: Cryptococcus neoformans species complex; COB1: Cytochrome b; COX1: Cytochrome c oxidase I; HEG: Homing endonuclease gene; LsrRNA: The large subunit of the ribosomal rRNA gene; MAT: Mating type; MATa: Mating type a; ND2: NADH dehydrogenase subunit 2; ND4: NADH dehydrogenase subunit 4; ND5: NADH dehydrogenase subunit 5; PCR-RFLP: Polymerase chain reaction - restriction fragment length polymorphisms; SD: Synthetic dextrose medium; YEPD: Yeast extract peptone dextrose medium

\section{Acknowledgements}

We thank Drs. Christina Hull and Joe Heitman for strains CHY618, CHY620, CHY647 and CHY648. We thank Heather Yoell for proof-reading the manuscript.

\section{Funding}

This project was funded by the Natural Science and Engineering Research Council (NSERC) of Canada, the Agricultural Science and Technology Innovation Program of the Chinese Academy of Agricultural Sciences (Grant\# CAAS-ASTIP-2015-IBFC) and the Elite Youth program of the Chinese Academy of Agricultural Sciences. We declare that the funding bodies had no role in the design of the study, in the collection, analysis, and interpretation of data, and in writing the manuscript.

Availability of data and materials

All the data supporting the findings are presented in the manuscript. For raw data, please contact author for data requests. 


\section{Authors' contributions}

ZY carried out the crosses and did sequence alignments. ZL and LY designed the primers and screened the parental mitochondrial genotypes. $Y Y, Y C, J C, Y L, C G$, and $L Z$ genotyped the progeny of the crosses. XS and $L G$ sequenced the COXI gene of representative progeny. ZY and JX conceived and designed the study. JX coordinated the drafting of the manuscript. All authors read and approved the final manuscript.

\section{Ethics approval and consent to participate}

Not applicable.

\section{Consent for publication}

Not applicable.

\section{Competing interests}

The authors declare that they have no competing interests.

\section{Publisher's Note}

Springer Nature remains neutral with regard to jurisdictional claims in published maps and institutional affiliations.

\section{Author details}

'Institute of Bast Fiber Crops, Chinese Academy of Agricultural Sciences, Changsha 410205, Hunan, China. ${ }^{2}$ Department of Biology, McMaster University, Hamilton, ON L8S 4K1, Canada.

\section{Received: 23 May 2018 Accepted: 10 July 2018}

\section{Published online: 17 July 2018}

\section{References}

1. Belfort M, Roberts RJ. Homing endonucleases: keeping the house in order. Nucleic Acids Res. 1997:25:3379-88.

2. Hurst GD, Werren $\mathrm{JH}$. The role of selfish genetic elements in eukaryotic evolution. Nat Rev Genet. 2001;2:597-606.

3. Goddard MR, Burt A. Recurrent invasion and extinction of a selfish gene. Proc Natl Acad Sci U S A. 1999:96:13880-5.

4. Belfort M, Perlman PS. Mechanisms of intron mobility. J Biol Chem. 1995; 270:30237-40.

5. Chevalier BS, Stoddard BL. Homing endonucleases: structural and functional insight into the catalysts of intron/intein mobility. Nucleic Acids Res. 2001; 29:3757-74.

6. Wu B, Hao W. Horizontal transfer and gene conversion as an important driving force in shaping the landscape of mitochondrial introns. G3: Genes, Genomes, Genetics. 2014;4:605-12.

7. Jacquier A, Dujon B. An intron-encoded protein is active in a gene conversion process that spreads an intron into a mitochondrial gene. Cell. 1985;41:383-94.

8. Gimble FS, Thorner J. Homing of a DNA endonuclease gene by meiotic gene conversion in Saccharomyces cerevisiae. Nature. 1992;357:301-6.

9. Gillham NW. Organelle Genes and Genomes. New York: Oxford University Press; 1994.

10. Birky CW Jr. Uniparental inheritance of mitochondrial and chloroplast genes: mechanisms and evolution. Proc Natl Acad Sci U S A. 1995;92:11331-8.

11. Birky CW Jr. The inheritance of genes in mitochondria and chloroplasts: laws, mechanisms, and models. Annu Rev Genet. 2001;35:125-48.

12. Wilson AJ. Xu J. Mitochondrial inheritance: diverse patterns and mechanisms with an emphasis on fungi. Mycology. 2012;3:158-66.

13. Xu J, Wang PF. Mitochondrial inheritance in basidiomycete fungi. Fungal Biol Rev. 2015;29:209-19.

14. Litter J, Keszthelyi A, Hamari Z, Pfeiffer I, Kucsera J. Differences in mitochondrial genome organization of Cryptococcus neoformans strains. Antonie Van Leeuwenhoek. 2005;88:249-55.

15. Xu J. Mitochondrial DNA polymorphisms in the human pathogenic fungus Cryptococcus neoformans. Curr Genet. 2002:41:43-7.

16. Zhang Y, Zhang S, Zhang G, Liu X, Wang C, Xu J. Comparison of mitochondrial genomes provides insights into intron dynamics and evolution in the caterpillar fungus Cordyceps militaris. Fungal Genet Biol. 2015;77:95-107.

17. Grun P. Cytoplasmic genetics and evolution. New York: Columbia University Press; 1976.
18. Law R, Hutson V. Intracellular symbionts and the evolution of uniparental cytoplasmic inheritance. Proc Biol Sci. 1992;248:69-77.

19. Coleman AW. Sex is dangerous in a world of potential symbionts or the basis of selection of uniparental inheritance. J Theor Biol. 1982;97:367-9.

20. Fedler M, Luh KS, Stelter K, Nieto-Jacobo F, Basse CW. The a2 mating-type locus genes lga2 and rga2 direct uniparental mitochondrial DNA (mtDNA) inheritance and constrain mtDNA recombination during sexual development of Ustilago maydis. Genetics. 2009;181:847-60.

21. Pfeifer A, Martin B, Kamper J, Basse CW. The mitochondrial LSU rRNA group II intron of Ustilago maydis encodes an active homing endonuclease likely involved in intron mobility. PLoS One. 2012;7:e49551.

22. Lemieux B, Turmel M, Lemieux C. Unidirectional gene conversions in the chloroplast of Chlamydomonas interspecific hybrids. Mol Gen Genet. 1988; 212:48-55.

23. Bussières JLC, Lee RW, Turmel M. Optional elements in the chloroplast DNAs of Chlamydomonas eugametos and C. moewusii: unidirectional gene conversion and co-conversion of adjacent markers in high-viability crosses. Curr Genet. 1996;30:356-65.

24. Yan Z, Xu J. Mitochondria are inherited from the MATa parent in crosses of the basidiomycete fungus Cryptococcus neoformans. Genetics. 2003;163: 1315-25.

25. Yan Z, Hull CM, Heitman J, Sun S, Xu J. SXII alpha controls uniparental mitochondrial inheritance in Cryptococcus neoformans. Curr Biol. 2004:14:R743-4.

26. Toffaletti DL, Nielsen K, Dietrich F, Heitman J, Perfect JR. Cryptococcus neoformans mitochondrial genomes from serotype a and D strains do not influence virulence. Curr Genet. 2004:46:193-204

27. Hull CM, Davidson RC, Heitman J. Cell identity and sexual development in Cryptococcus neoformans are controlled by the mating-type-specific homeodomain protein Sxi1alpha. Genes Dev. 2002;16:3046-60.

28. Yan Z, Li X, Xu J. Geographic distribution of mating type alleles of Cryptococcus neoformans in four areas of the United States. J Clin Microbiol. 2002:40:965-72.

29. Hausner G. Introns, Mobile Elements, and Plasmids. Sackville New Brunswick Canada: Springer Berlin Heidelberg; 2012

30. Baptista-Ferreira UL, Economou A, Casselton LA. Mitochondrial genetics of Coprinus: recombination of mitochondrial genomes. Curr Genet. 1983;7:405-7.

31. Barroso G, Labarere J. Genetic evidence for nonrandom sorting of mitochondria in the basidiomycete Agrocybe aegerita. Appl Environ Microbiol. 1997;63:4686-91.

32. Matsumoto T, Fukumasa-Nakai Y. Mitochondrial DNA inheritance in sexual crosses of Pleurotus ostreatus. Curr Genet. 1996;30:549-52.

33. Collins RA, Saville BJ. Independent transfer of mitochondrial chromosomes and plasmids during unstable vegetative fusion in Neurospora. Nature. 1990; 345:177-9.

34. $\mathrm{Xu}$ J. The inheritance of organelle genes and genomes: patterns and mechanisms. Genome. 2005;48:951-8.

35. Muller HJ. The relation of recombination to mutational advance. Mutat Res. 1964;106:2-9

36. $\mathrm{Xu}$ J. Genotype-environment interactions of spontaneous mutations for vegetative fitness in the human pathogenic fungus Cryptococcus neoformans. Genetics. 2004;168:1177-88.

37. Christie JR, Beekman M. Selective sweeps of mitochondrial DNA can drive the evolution of uniparental inheritance. Evolution. 2017:71:2090-9.

38. Christie JR, Schaerf TM, Beekman M. Selection against heteroplasmy explains the evolution of uniparental inheritance of mitochondria. PLoS Genet. 2015: 11:e1005112.

39. Boynton JE, Harris EH, Burkhart BD, Lamerson PM, Gillham NW. Transmission of mitochondrial and chloroplast genomes in crosses of Chlamydomonas. Proc Natl Acad Sci U S A. 1987;84:2391-5.

40. Bendich AJ. DNA abandonment and the mechanisms of uniparental inheritance of mitochondria and chloroplasts. Chromosom Res. 2013;21:287-96.

41. Williamson D. The curious history of yeast mitochondrial DNA. Nat Rev Genet. 2002;3:475-81.

42. MacAlpine DM, Kolesar J, Okamoto K, Butow RA, Perlman PS. Replication and preferential inheritance of hypersuppressive petite mitochondrial DNA. EMBO J. 2001:20:1807-17.

43. Kwon-Chung KJ, Edman JC, Wickes BL. Genetic association of mating types and virulence in Cryptococcus neoformans. Infect Immun. 1992:60:602-5.

44. Mead ME, Stanton BC, Kruzel EK, Hull CM. Targets of the sex inducer homeodomain proteins are required for fungal development and virulence in Cryptococcus neoformans. Mol Microbiol. 2015;95:804-18. 
45. Belfort M, Bonocora RP. Homing endonucleases: from genetic anomalies to programmable genomic clippers. Methods Mol Biol. 2014;1123:1-26.

46. Windbichler N, Menichelli M, Papathanos PA, Thyme SB, Li H, Ulge UY. A synthetic homing endonuclease-based gene drive system in the human malaria mosquito. Nature. 2011;473:212-5.

47. Haugen P, Bhattacharya D. The spread of LAGLIDADG homing endonuclease genes in rDNA. Nucleic Acids Res. 2004;32:2049-57.

Ready to submit your research? Choose BMC and benefit from:

- fast, convenient online submission

- thorough peer review by experienced researchers in your field

- rapid publication on acceptance

- support for research data, including large and complex data types

- gold Open Access which fosters wider collaboration and increased citations

- maximum visibility for your research: over $100 \mathrm{M}$ website views per year

At $\mathrm{BMC}$, research is always in progress.

Learn more biomedcentral.com/submissions 